\title{
Cyclosporin effect on noradrenaline release from the sympathetic nervous endings of rat aorta
}

\author{
Paula Tavares, C.A. Fontes Ribeiro, F. Teixeira* \\ Institute of Pharmacology and Experimental Therapeutics, Faculty of Medicine, University of Coimbra, 3049 Coimbra codex, Portugal
}

Accepted 30 September 2002

\begin{abstract}
Arterial hypertension is one of the main side effects of cyclosporin treatment and seems to be due to activation of the sympathetic nervous system. Some authors hypothesized that cyclosporin may act on the sympathetic nervous endings increasing catecholamine release, in agreement with our previous works which demonstrated an increase in rat plasma catecholamine levels after $30 \mathrm{mg} / \mathrm{kg}$ per day cyclosporin treatment for 7 weeks. Therefore, the aim of this work was to study the cyclosporin mechanism responsible for that increase in plasma catecholamines. Male Wistar rats were used. Noradrenaline release was performed in vitro experiments after loading rat aorta abdominal segments with ${ }^{3} \mathrm{H}$-noradrenaline $\left({ }^{3} \mathrm{H}-\mathrm{NA}\right)$. The release of ${ }^{3} \mathrm{H}-\mathrm{NA}$ was measured after electrical stimulation in the presence of $10^{-6} \mathrm{M}$ cyclosporin. In another set of experiments electrical stimulation was replaced by a pulse addition of cyclosporin $\left(10^{-6} \mathrm{M}\right)$. Another group of rats was treated with $30 \mathrm{mg} / \mathrm{kg}$ per day cyclosporin for 7 weeks and catecholamine contents in aorta abdominal segments and adrenals were measured by high performance liquid chromatography system with electrochemical detection (HPLC-ECD). An increase in the ${ }^{3} \mathrm{H}-\mathrm{NA}$ release was observed in both types of in vitro experiments. Since cocaine abolished these cyclosporin effects, the obtained results suggest that cyclosporin may act on the catecholamine transporter across the membrane. In addition, after the 7 weeks of cyclosporin treatment, a significant decrease in catecholamine aorta contents was verified but in adrenals there was no difference regarding to controls. However, the dopamine synthesis/degradation ratio measured by the DA/DOPAC ratio suggests an increase in dopamine synthesis. These facts are in agreement with the enhanced plasma catecholamine levels and with the hypothesis of tissue catecholamine depletion. However, they do not explain the increase in plasma adrenaline levels, since adrenaline is a reflex of adrenal activity. The synthesized dopamine in adrenals seems to be unable to reach vesicles and to be metabolized in adrenaline. The observed decrease in HVA adrenal levels may be a consequence of extraneuronal uptake inhibition or inhibition by cyclosporin of the direct $o$-methylation of DOPAC.

In conclusion, our results suggest that cyclosporin increases catecholamine release from the sympathetic nervous endings by a tyraminelike effect, i.e. by acting directly on the catecholamine transporter of the membrane.
\end{abstract}

(C) 2002 Elsevier Science Ltd. All rights reserved.

Keywords: Cyclosporin; Noradrenaline release; Rat aorta; Sympathetic nervous system

\section{Introduction}

Cyclosporin is an immunosuppressive drug that has considerably improved the survival of transplant patients in recent years. However, several side effects have been associated with cyclosporin treatment, such as hypertension, nephrotoxicity and neurotoxicity. The possible mechanisms underlying cyclosporin-induced hypertension were reviewer by Sander and Victor [1]. Nevertheless, none of the hypothesis is out of controversy. Thus, the only fact that is in agreement with all of them is that cyclosporin changes vascular reactivity. Concerning noradrenaline and

\footnotetext{
${ }^{*}$ Corresponding author. Tel.: +351-239857700; fax: +351-239823236.

E-mail address: fredjt@ci.uc.pt (F. Teixeira).
}

phenilephrine-induced contraction, cyclosporin seems to increase [2-5] or decrease [6-8] vessel contraction. To explain these facts Xue et al. [2] hypothesize that after an inhibition of the $\alpha_{2}$, adrenoceptors cyclosporin increases contraction by an increase of noradrenaline release from the sympathetic nervous endings. Also Yaris and Tuncer in 1995 [3], indicated cyclosporin as a possible $\alpha$ adrenoceptors inhibitor, after the observation that cyclosporin increased noradrenaline levels more than those obtained by electrical stimulation. Moreover, studies on plasma catecholamine levels in human patients and animals treated with cyclosporin have provided controversial results. In this sense, Van den Dopel et al. [9] and Sehested et al. [10] found no changes in plasma noradrenaline levels in patients with kidney transplant and hypertension undergoing treatment 
with cyclosporin. In contrast, also in humans and under similar conditions, Crum et al. [11] observed an increase in plasma adrenaline and noradrenaline levels. Experiments in cyclosporin-treated rats have also afforded conflicting results. In a study following the administration of 5, 10 and $20 \mathrm{mg} / \mathrm{kg}$ per day cyclosporin for 3 weeks, Gerkens [12] did not find any changes in plasma catecholamine levels. However, Duruibe et al. [13] described an increase in noradrenaline and adrenaline levels but not of dopamine. More recently, our research group observed a significant increase in rat plasma catecholamine levels (noradrenaline, adrenaline and dopamine) after 7 weeks of treatment with $30 \mathrm{mg} / \mathrm{kg}$ per day cyclosporin-Sandimmune Neoral ${ }^{\circledR}$ [14]. As expected, these results were directly correlated with cyclosporin-induced hypertension. The observed effect of cyclosporin on plasma catecholamine levels may be due to alterations in the sympathetic nerve endings of blood vessels, promoted by the immunosuppressive drug. In the light of these findings, the goal of the present work was to study the effect of cyclosporin in the noradrenaline release from the sympathetic nerve endings of blood vessels. We also studied the effect of cyclosporin treatment on the catecholamine contents of rat abdominal aorta and adrenals in order to contribute for a possible explanation of the changes in plasma catecholamine levels and subsequent hypertension.

\section{Material and methods}

Male Wistar rats (weighing 300-350g at the beginning of experiments) were housed four to five per cage under a $12 \mathrm{~h} \mathrm{light/dark}$ cycle in a room with controlled temperature $\left(22 \pm 1^{\circ} \mathrm{C}\right)$, humidity $(50 \pm 10 \%)$ with food and water available ad libitum. The experimental animals were handled as carefully as possible in order to minimize environmental stress. Animal experiments were carried out in agreement with the European Convention on Animal Care, and the whole research project in which these studies are included received the approval of the Portuguese National Foundation for Science and Technology.

\subsection{Experimental procedure}

The animals were randomized into two groups: Group I was treated with $30 \mathrm{mg} / \mathrm{kg}$ cyclosporin and Group II received only the solvent (control group). Rats were treated daily with cyclosporin for a period of 7 weeks by oral administration. The cyclosporin was the commercial formulation (Sandimmune Neoral ${ }^{\circledR}$ ) dissolved in orange juice as indicated for human treatment. Both groups received the same volume of the drug or solvent $(0.5 \mathrm{ml})$.

The cyclosporin dose used in vivo induced a blood concentration similar to that found in humans, as discussed before [15]. Thus, cyclosporin blood levels measured in our treated rats were $1.76 \pm 0.06 \mu \mathrm{M}$ and $0.43 \pm 0.11 \mu \mathrm{M}$ at 14 and $36 \mathrm{~h}$, respectively, after the last administration of $30 \mathrm{mg} / \mathrm{kg}$ per day cyclosporin. Moreover, the $10^{-6} \mathrm{M}$ cyclosporin corresponds approximately to maximal plasma values attained in human pharmacokinetics $[16,17]$. Based on these results, a $10^{-6} \mathrm{M}$ cyclosporin concentration was used in all in vitro experiments.

\subsection{Dissection and determination of monoamines}

After the 7 weeks cyclosporin treatment the animals were sacrificed by pentobarbital sodium overdose. The abdominal segment of aorta as well as adrenals were rapidly removed and cleaned from surrounding tissue. Small segments of abdominal aorta and adrenals were immediately placed into $0.1 \mathrm{M}$ perchloric acid $\left(\mathrm{HClO}_{4}\right)$ solution and kept overnight. Aliquots of the extract were directly injected in a high performance liquid chromatography system with electrochemical detection (HPLC-ECD).

The chromatographic system consisted of a Gilson Applied Chromatographic System with a 305 model pump and a 231 injection valve model, with a $50 \mu 1$ loop. A Biophase ODS RP18 analytical column $(250 \times 4.6, \varnothing=5 \mu \mathrm{m}$; Bioanalytical Systems Inc., USA) was used and separation was made possible by using an isocratic solvent system consisting of an acetate-citrate buffer (sodium acetate $0.1 \mathrm{M}$, citric acid $0.1 \mathrm{M}$ ), containing sodium octane sulfonate $(0.5 \mathrm{mM}), \mathrm{Na}_{2}$ EDTA $(0.15 \mathrm{mM})$, dibutylamine $(1 \mathrm{mM})$ and $10 \%$ methanol. A flow rate of $1 \mathrm{ml} / \mathrm{min}$ was maintained and detection of the chromatographed catecholamines achieved by using a 141 Gilson electrochemical detector model $(650 \mathrm{mV})$.

\subsection{Release of ${ }^{3} \mathrm{H}$-noradrenaline}

\subsubsection{Incubation with ${ }^{3} H$-noradrenaline}

The strips of abdominal aorta were immersed in a Krebs physiologic solution of the following composition (which was also used for superfusion); mmol/l: $118.67 \mathrm{NaCl} ; 5.36$ $\mathrm{KCl} ; 1.90 \mathrm{CaCl}_{2} ; 0.67 \mathrm{NaH}_{2} \mathrm{PO}_{4} ; 0.57 \mathrm{NaHCO}_{3} ; 11.1$ glucose; $\mathrm{pH}$ 7.4. To avoid oxidation of noradrenaline, $\mathrm{Na}_{2}$ EDTA 0.027 and ascorbic acid $0.057 \mathrm{mmol} / \mathrm{l}$ were added to the solution which was maintained at $37{ }^{\circ} \mathrm{C}$ and bubbled with $95 \%$ $\mathrm{O}_{2}$ and $5 \% \mathrm{CO}_{2}$. Tissues were first exposed for $30 \mathrm{~min}$ to pargyline $(1 \mathrm{mmol} / \mathrm{l})$ to inhibit monoamine oxidase (MAO), and then incubated with ${ }^{3} \mathrm{H}$-noradrenaline $\left({ }^{3} \mathrm{H}-\mathrm{NA}\right.$ ) (for $1 \mathrm{~h}$ in small beakers containing $5 \mathrm{ml}$ medium with $(-)-{ }^{3} \mathrm{H}-\mathrm{NA}$ $(0.23 \mu \mathrm{mol} / \mathrm{l})$.

\subsubsection{Spontaneously and electrically-induced release of ${ }^{3} \mathrm{H}$-noradrenaline}

After incubation, the tissues were transferred into $1 \mathrm{ml}$ glass chambers and perfused with ${ }^{3} \mathrm{H}-\mathrm{NA}$-free medium from bottom to top at a rate of $0.8 \mathrm{ml} / \mathrm{min}$. Two protocols were performed.

2.3.2.1. Protocol 1: electrically-induced ${ }^{3} H$-noradrenaline release. After a washout period (of $75 \mathrm{~min}$ ), the perfusion 
fluid was collected continuously, in 5-min samples, from $t=75 \mathrm{~min}$ to $t=210 \mathrm{~min}(t=0$ being the start of perfusion). In order to assess the basal and electrically evoked outflow of ${ }^{3} \mathrm{H}-\mathrm{NA}, 5$-min period of transmural stimulation ( $3 \mathrm{~Hz}, 1 \mathrm{~ms}, 100 \mathrm{~V}, 300$ pulses, stimulator SD9B, Grass Instruments, MA, USA) [from $t=110 \mathrm{~min}$ to $t=115 \mathrm{~min}$ (S1); from $t=130 \mathrm{~min}$ to $t=135 \mathrm{~min}$ (S2); from $t=150 \mathrm{~min}$ to $t=155 \mathrm{~min}(\mathrm{~S} 3)$; from $t=170 \mathrm{~min}$ to $t=175 \mathrm{~min}$ (S4); from $t=190 \mathrm{~min}$ to $t=195 \mathrm{~min}$ (S5)] were performed. These experiments were done in the presence and absence of cyclosporin $\left(10^{-6} \mathrm{M}\right)$ and/or cocaine $\left(7.5 \times 10^{-6} \mathrm{M}\right)$. The drugs were placed into the bath $10 \mathrm{~min}$ before S3 and maintained during the S4 and S5 periods. Aliquots of each fraction collected were added to scintillation fluid (1:3), and the radioactivity measured in a Packard 2000 spectrometer provided with dpm correction. At the end of the experiment the segments were removed from the chambers, dried and heighted, and the amount of ${ }^{3} \mathrm{H}-\mathrm{NA}$ retained measured. The stimulation-evoked overflow was obtained by subtraction of the estimated basal outflow from the total overflow in stimulation and poststimulation samples. The evoked overflow was expressed as fractional release per shock in relation to $\mathrm{S} 2$. To obtain the ${ }^{3} \mathrm{H}-\mathrm{NA}$ that was retained in the vessel, dpm values were divided by segments weight.

\subsubsection{Protocol 2: spontaneously release of ${ }^{3} \mathrm{H}$-noradre-} naline. After a washout period (of $75 \mathrm{~min}$ ), the perfusion fluid was collected continuously, in 5-min samples, from $t=75 \mathrm{~min}$ to $t=150 \mathrm{~min}(t=0$ being the start of perfusion). The drugs were added from $t=110 \mathrm{~min}$ to $t=115 \mathrm{~min}(\mathrm{E} 1)$ and from $t=130 \mathrm{~min}$ to $t=135 \mathrm{~min}$ (E2) (cyclosporin $10^{-6} \mathrm{M}$; cocaine $7.5 \times 10^{-6} \mathrm{M}$; tyramine $\left.5 \times 10^{-5} \mathrm{M}\right)$. Aliquots of each fraction were added to the scintillation fluid (1:3), and the radioactivity measured in a Packard 2000 spectrometer provided with dpm correction.

At the end of the experiment the segments were removed from the chambers, dried and heighted and measured the amount of ${ }^{3} \mathrm{H}-\mathrm{NA}$ retained.

After correction by vessel weight, values in each stimulation point (E1 and E2) were calculated according to the expression:

$$
\begin{aligned}
& \% \text { of }{ }^{3} \mathrm{H}-\mathrm{NA} \text { release in } \mathrm{E} \\
& =\left(\begin{array}{c}
\frac{\left.{ }^{3} \mathrm{H}-\mathrm{NA} \text { (released in } \mathrm{E}\right)}{\mathrm{H}-\mathrm{NA} \text { (total released) }}+{ }^{3} \mathrm{H}-\mathrm{NA} \text { (retained in the segment) }
\end{array}\right) \times 100
\end{aligned}
$$

The amount of ${ }^{3} \mathrm{H}-\mathrm{NA}$ retained in the vessel was calculated according to the following expression:

$$
\begin{aligned}
& \% \text { of }{ }^{3} \mathrm{H}-\mathrm{NA} \text { in the vessel } \\
& \quad=\left(\frac{\mathrm{dpm} / \text { vessel weight }}{\text { total released }+ \text { total in the vessel }}\right) \times 100
\end{aligned}
$$

\subsection{Measurement of the radioactivity retained in the vessel}

The vascular segments were heat $\left(100{ }^{\circ} \mathrm{C}\right.$ in a dry bath) with $200 \mu \mathrm{l} \mathrm{H}_{2} \mathrm{O}_{2}$ (30 vol.) plus $200 \mu \mathrm{HClO}_{4}, 30 \%$ during $15 \mathrm{~min}$. To the solubilized samples scintillation fluid was added, and the radioactivity measured in a Packard 2000 spectrometer provided with dpm correction.

In both protocols using ${ }^{3} \mathrm{H}-\mathrm{NA}$ a HPLC-ECD control was performed in order to determine that in the final of the experiments we, in fact, measured noradrenaline and not other substances like noradrenaline metabolites.

\subsection{Preparation of cyclosporin}

The majority of authors used ethanol to dissolve cyclosporin. However, this solvent could have some undesired effects. To avoid these pitfalls, we used dimethyl sulfoxide (DMSO) as solvent. In all control experiments DMSO was also added. The possible morphological-induced alterations of this cyclosporin solution were tested in smooth muscle cell cultures. DMSO (in the percentage used) showed no effects on cell morphology or viability (data not showed).

\subsection{Chemicals}

Cyclosporin and Sandimmune $\mathrm{Neoral}^{\circledR}$ were supplied by Novartis Farma, Portugal. $\left[{ }^{3} \mathrm{H}\right]-\mathrm{NA}$ (specific activity = $12 \mathrm{Ci} / \mathrm{mmol}$ ) was obtained from Amersham (USA). The scintillation fluid (UNIVERSOL) was obtained from ICN (Costa Mesa). All the other chemicals were obtained from Sigma, St. Louis, MO, USA.

\subsection{Statistics}

Fisher's protected least significant difference (PLSD) test preceded by one-way ANOVA were applied. Probability levels of less than 0.05 were regarded as statistically significant.

\section{Results}

In a previous published work we demonstrated that an increase in plasma catecholamine levels was observed after cyclosporin administration [14]. In those animals the increase in plasma catecholamine levels was correlated with an increase in blood pressure. Moreover, cyclosporin induced arterial hypertension after the first week of treatment. Since we hypothesize that the increase in plasma catecholamine levels was due to a cyclosporin action on the vascular sympathetic nervous endings, the aorta catecholamine contents were measured in the present work. After 7 weeks of $30 \mathrm{mg} / \mathrm{kg}$ per day cyclosporin treatment, a significant decrease in catecholamine contents (noradrenaline, adrenaline and dopamine) was observed in abdominal aorta segments (Fig. 1). To further understand the cyclosporin effect on 


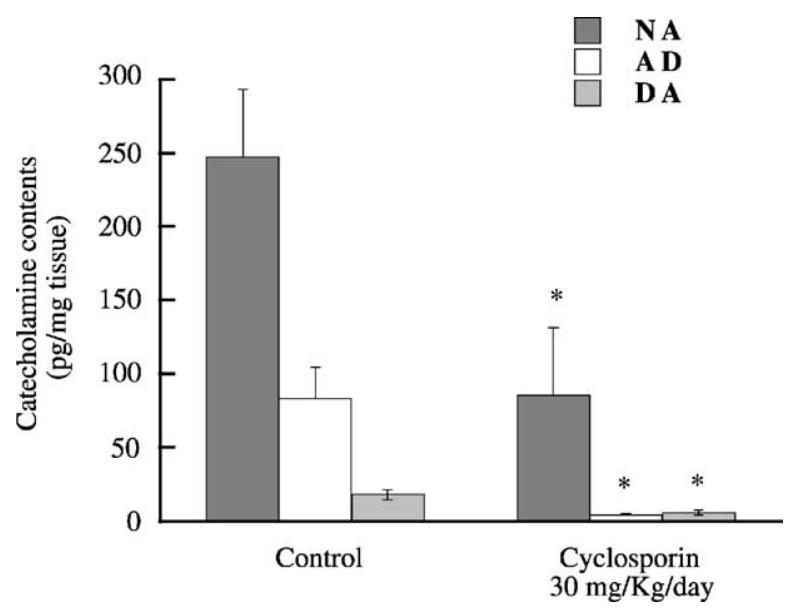

Fig. 1. Catecholamine contents in rat abdominal aorta segments after 7 weeks of treatment with solvent (control) $(n=10)$ or with CsA $30 \mathrm{mg} / \mathrm{kg}$ per day $(n=12)$. Catecholamines [noradrenaline (NA), adrenaline (AD) or dopamine (DA)] were determined by HPLC-ECD. Data shown are means \pm S.E.M. ${ }^{*} P<0.05$ related to control. vascular noradrenaline release, ${ }^{3} \mathrm{H}-\mathrm{NA}$ loading experiments were performed. Thus, ${ }^{3} \mathrm{H}-\mathrm{NA}$ was released by electrical stimulation, in the presence of cyclosporin, or by cyclosporin itself. Both ${ }^{3} \mathrm{H}-\mathrm{NA}$ release protocols showed that cyclosporin increases ${ }^{3} \mathrm{H}-\mathrm{NA}$ release. After electrical stimulation, cyclosporin increased both $\mathrm{S} 3 / \mathrm{S} 2$ and $\mathrm{S} 4 / \mathrm{S} 2$ release fraction ratios, without changes of the $\mathrm{S} 5 / \mathrm{S} 2$ ratio (Table 1). When cocaine was added to the organ bath, the calculated fraction ratios became similar to controls. Cocaine alone, as expected, show a slight increase in ${ }^{3} \mathrm{H}-\mathrm{NA}$ release when compared to control (Table 1 ). In addition, aorta ${ }^{3} \mathrm{H}-\mathrm{NA}$ contents, after the electrically-induced efflux, were decreased, in agreement with the described release results (Table 1). When the electrical stimulation was replaced by the direct cyclosporin addition to the organ bath ${ }^{3} \mathrm{H}-\mathrm{NA}$ release also increased (Fig. 2). Once more, cocaine abolished this cyclosporin effect. These results, from both protocols, may indicate a cyclosporin tyramine-like effect. In fact, when cyclosporin was replaced by tyramine results showed

Table 1

Electrically-induced release of ${ }^{3} \mathrm{H}$-noradrenaline from rat aorta segments

\begin{tabular}{lccc}
\hline Release fraction ratio & Control & Control + cocaine & Cyclosporin \\
\hline S3/S2 & $0.847 \pm 0.042$ & $0.974 \pm 0.087$ & $1.109 \pm 0.093^{*}$ \\
S4/S2 & $0.802 \pm 0.031$ & $0.852 \pm 0.073$ & $0.875 \pm 0.001^{\#}$ \\
S5/S2 & $0.656 \pm 0.220$ & $0.894 \pm 0.109$ & $0.817 \pm 0.049$ \\
Aorta (dpm/mg tissue) & $4305.6 \pm 157.9$ & $3402.5 \pm 165.8^{*}$ & $0.795 \pm 0.084$ \\
\hline
\end{tabular}

Cyclosporin and/or cocaine were added before S3 and remained for all the experiment. In each period of stimulation the release fraction was calculated according to the expression indicated in Section 2. Values represent mean \pm S.E.M. of five independent experiments performed in triplicate.

* $P<0.05$ related to control.

\# $P<0.05$ related to cyclosporin.

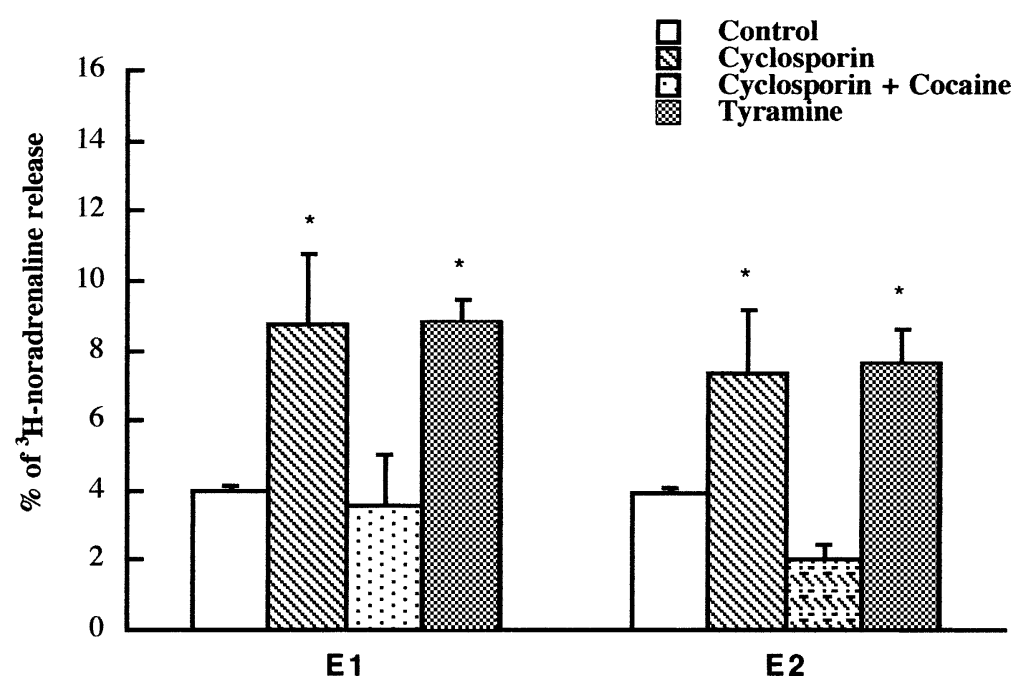

Fig. 2. Drug-induced release of ${ }^{3} \mathrm{H}$-noradrenaline from rat abdominal aorta segments. Cyclosporin and/or cocaine were added in E1 and E2, except in control (only with the solvent) and experiments with tyramine. In each period of stimulation the percent of ${ }^{3} \mathrm{H}$-noradrenaline released was calculated according to the expression indicated in Section 2. Values represent mean \pm S.E.M. of five independent experiments performed in triplicate. ${ }^{*} P<0.05$ related to control of five experiments. ${ }^{\#} P<0.05$ related to cyclosporin. 


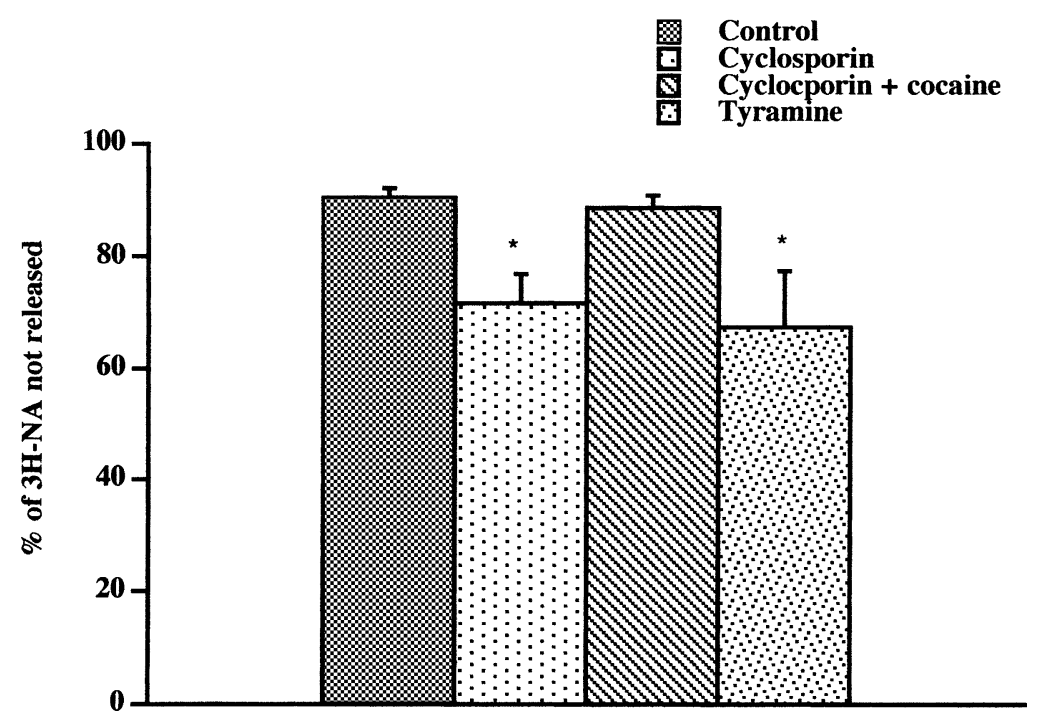

Fig. 3. Abdominal aorta contents of ${ }^{3} \mathrm{H}$-noradrenaline (percent of ${ }^{3} \mathrm{H}$-noradrenaline not released) after the experiments indicated in Fig. 2. Values were calculated according to the expression indicated in Section 2. Values represent mean \pm S.E.M. of five independent experiments performed in triplicate. ${ }^{*} P<0.05$ related to control of five experiments.

a similar profile (Fig. 2). Moreover, the accumulated percent of ${ }^{3} \mathrm{H}-\mathrm{NA}$ in aorta segments reinforces this behavior (Fig. 3). All these results seem to suggest that cyclosporin increases ${ }^{3} \mathrm{H}-\mathrm{NA}$ release from the sympathetic nervous endings.

The ${ }^{3} \mathrm{H}-\mathrm{NA}$ in vitro studies could explain the increase in noradrenaline plasma levels and the decrease in vascular noradrenaline contents of cyclosporin-treated rats. However, the increased adrenaline plasma levels may only be explained by cyclosporin-induced changes in adrenals. When the adrenal catecholamine contents were measured, from cyclosporin-treated animals, no significant changes were observed when compared to controls (Fig. 4). However, 3,4-dihydroxyphenylacetic acid (DOPAC) values significantly increased whereas homovanillic acid (HVA) values

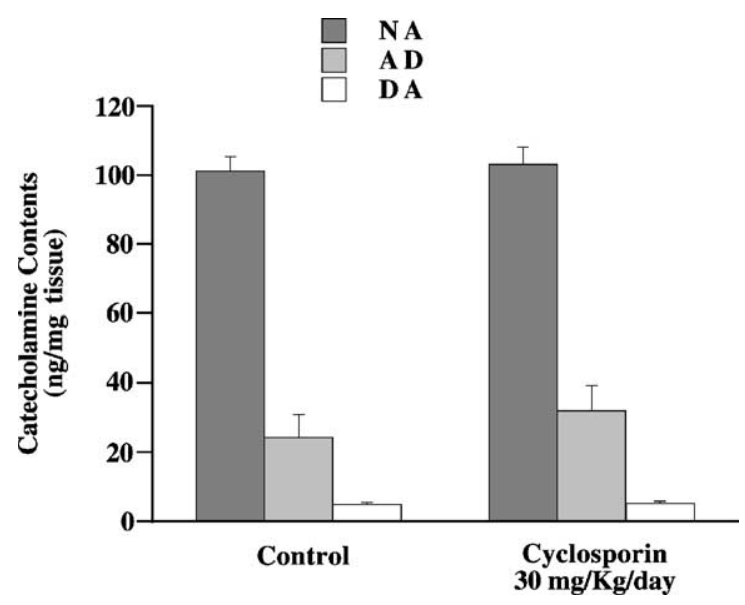

Fig. 4. Adrenal catecholamine contents in the rat after 7 weeks of treatment with vehicle (control) $(n=10)$ or with CsA $30 \mathrm{mg} / \mathrm{kg}$ per day $(n=12)$. Catecholamines [noradrenaline (NA), adrenaline (AD) and dopamine (DA)] were determined by HPLC-ECD. Data shown are means \pm S.E.M. decreased (Fig. 5). The sum DOPAC + DA (indicator of synthesis) as well as the DOPAC/DA ratio (indicator of metabolization) showed a significant increase when compared to control (Table 2).

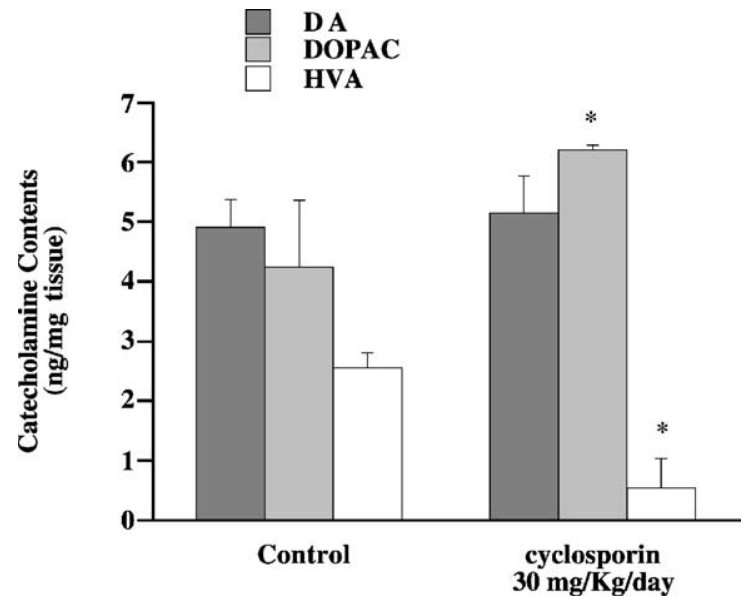

Fig. 5. Adrenal dopamine and dopamine metabolite contents in rats after 7 weeks of treatment with vehicle (control) $(n=10)$ or with CsA $30 \mathrm{mg} / \mathrm{kg}$ per day $(n=12)$. Catecholamines [dopamine (DA), 3,4-dihydroxyphenylacetic acid (DOPAC) and homovanillic acid (HVA)] were determined by HPLC-ECD. Data shown are means \pm S.E.M. ${ }^{*} P<0.05$ (vs. corresponding control).

Table 2

Indicators of dopamine (DA) synthesis and degradation

\begin{tabular}{lll}
\hline & Control $(n=10)$ & $\begin{array}{l}\text { Cyclosporin } 30 \mathrm{mg} / \mathrm{kg} \\
\text { per day }(n=12)\end{array}$ \\
\hline DOPAC/DA $(\times 100)$ & $89.20 \pm 11.30$ & $124.40 \pm 13.20^{*}$ \\
DOPAC + DA $(\mathrm{ng} / \mathrm{mg})$ & $9.150 \pm 0.491$ & $11.361 \pm 0.939^{*}$ \\
\hline
\end{tabular}

Synthesis was evaluated by the sum of DOPAC and DA; degradation was evaluated by the DOPAC/DA ratio. Values represent mean \pm S.E.M.

${ }^{*} P<0.05$ related to control. 


\section{Discussion}

Several mechanisms, including the activation of the sympathetic nervous system, have been proposed to explain the cyclosporin-induced hypertension. However, like other explanations this hypothesis is also not free of controversy. For instance, Stein et al. [18] showed in humans an impaired vasodilatation rather than sympathetic activation or enhanced vasoconstriction, which may be an important mechanism for the alterations of the vascular tone that occurs after long-term cyclosporin administration. Nevertheless, behind all the controversy that surrounds cyclosporin-induced hypertension there is one common point: a change in vascular reactivity in response to several agents, namely noradrenaline. However, the way of this change is also contradictory in the literature. In response to noradrenaline or phenylephrine cyclosporin seems to increase [2-5] or decrease [6-8] vascular contraction. Several possible mechanisms have been proposed for the increased sympathetic nerve activity: direct release of the transmitter induced by cyclosporin from sympathetic nerves [2] or potentiation of responses to nerve stimulation $[19,20]$. According to the hypothesis purposed by Xue et al. [2], our results also showed an increase in the noradrenaline release. Since aorta segments are poorly enervated, the experiments were performed with exogenous noradrenaline $\left({ }^{3} \mathrm{H}-\mathrm{NA}\right)$. In our experiments, after electrical stimulation the presence of cyclosporin increased ${ }^{3} \mathrm{H}-\mathrm{NA}$ release and decreased ${ }^{3} \mathrm{H}-\mathrm{NA}$ contents in the vascular segments. It seems that cyclosporin may increase the noradrenaline release by acting through the membrane noradrenaline transporter. To test this hypothesis, the ${ }^{3} \mathrm{H}-\mathrm{NA}$ studies were performed in the presence of cocaine. The results showed that in the presence of cocaine cyclosporin was unable to induce any increase on ${ }^{3} \mathrm{H}-\mathrm{NA}$ release. As expected, cocaine by itself increases ${ }^{3} \mathrm{H}-\mathrm{NA}$ release. Thus, these results seem to suggest that cyclosporin may have tyramine-like effect. When the experiments were repeated without electrical stimulation but with addiction of cyclosporin we verified the same behavior, i.e. an increase in ${ }^{3} \mathrm{H}-\mathrm{NA}$ release. Once more this was similar to the tyramine effect. So, as it happens with tyramine or amphetamines, cyclosporin may also use the uptake 1 to enter into the axoplasm. The hypothesis that cyclosporin could also enter by passive diffusion through the membrane (like tyramine in high concentrations) is not probable since cocaine abolish the cyclosporin effect. Once in the cytoplasm, cyclosporin enters axoplasmic vesicles and like tyramine or amphetamines decreases the vesicle $\mathrm{pH}$ gradient [21]. This will decrease ${ }^{3} \mathrm{H}-\mathrm{NA}$ protonation and increase ${ }^{3} \mathrm{H}-\mathrm{NA}$ release to the cytoplasm and rapidly from this space to outside. Therefore, cyclosporin seems to act more upon the noradrenaline transporter than in the plasma membrane, which is in agreement with contraction studies in response to potassium where cyclosporin has no effect (unpublished data from the authors). On the contrary, observations of Lamb and Webb [19], in rat tail arteries, indicate that $8.3 \times 10^{-6} \mathrm{M}$ cyclosporin appears to be acting at the level of the plasma membrane potential. The decrease in the membrane potential increases both release of noradrenaline from adrenergic nerve ending and smooth muscle responses to applied noradrenaline. In our study, electrical stimulation increases nervous endings stimulation with a consequent enhancement of noradrenaline transport activity, as well as the probable cyclosporin tyramine-like effect. In addition, cyclosporin has no effect on potassium-induced contraction (data not shown).

In the light of our results, after repeated cyclosporin treatment vessels were expected to be depleted of catecholamines. Few works have addressed the effect of cyclosporin on catecholamine tissue contents. In 1995, Pestana et al. [22] reported an accumulation of DA and DOPAC in the rat kidney tissue after 14 days of treatment with $50 \mathrm{mg} / \mathrm{kg}$ per day cyclosporin. In the present work, after 7 weeks of cyclosporin treatment a decrease of the abdominal aorta catecholamine contents were observed. These data are in agreement with our hypothesis that cyclosporin may have a tyramine-like effect. This effect could also partially justify the increase in plasma catecholamines previously reported [14]. The increase in plasma adrenaline found before [14] is difficult to explain with this hypothesis, since plasma adrenaline levels reflect the amounts of adrenaline synthesized and released by adrenal glands. We found no significant changes in adrenaline contents in the adrenals of cyclosporin-treated animals as compared with the controls. The same results in adrenals were found as regards the other catecholamines assayed (noradrenaline and dopamine). Despite this, on analyzing the dopamine synthesis/degradation ratio, as measured by dopamine metabolite levels, and the relations DOPAC + DA and DOPAC/DA some differences were observed. The results obtained in the group of rats treated with $30 \mathrm{mg} / \mathrm{kg}$ per day cyclosporin suggest an increase in dopamine synthesis. For some reason, however, this dopamine (or at least not all the dopamine synthesized) was unable to enter neuronal vesicles to be metabolized into noradrenaline and adrenaline. Some of it was converted into DOPAC outside the vesicles leading to the verified increase in DOPAC levels. These findings are in agreement with the results obtained by Pestana et al. [22] in rat kidney tissue after $50 \mathrm{mg} / \mathrm{kg}$ per day cyclosporin treatment. According to our results, it would appear that vesicles have some difficulty in incorporating dopamine. A possible reason could be the hypothesis discussed above that cyclosporin may have a direct effect on catecholamine transport across membranes. Moreover, in the adrenals of this group of rats a decrease in HVA levels was also observed, suggesting that this concentration of cyclosporin might also interfere in the conversion of DOPAC to HVA, inhibiting extraneuronal uptake and subsequent metabolization by COMT (catechol-o-methyltransferase), or directly inhibiting DOPAC $o$-methylation.

In spite of the absence of differences in adrenals catecholamine contents we could not exclude the fact that cyclosporin could increase both adrenal catecholamine 
synthesis and release, since the sum of DA and DOPAC were increased.

It was been hypothesized that cyclosporin-induced hypertension could be caused by renal and other sub-diaphragmatic afferent activation, which reflexively activates efferent sympathetic, nerve activity and therefore causes neurogenic vasoconstriction $[23,24]$. This could be also be an explanation for our in vivo results. However, we also demonstrated that more than this effect on the activation of sympathetic nerve activity, cyclosporin has also a direct effect over the sympathetic nervous endings.

In conclusion, our results suggest that cyclosporin increase the noradrenaline release from sympathetic nervous endings, probably by interfering on the catecholamine transport across membrane rather than by an action on the plasma membrane potential. This fact may explain the increase in catecholamine plasma levels and the consequent arterial hypertension, as well as the increase in adrenal DOPAC.

\section{Acknowledgements}

We are grateful to Novartis Inc. for their kind collaboration in this project. This work was supported by a grant from the Portuguese National Foundation for Science and Technology (Praxis Program)—PRAXIS/PSAU/C/SAU/57/96.

\section{References}

[1] Sander M, Victor RG. Hypertension after cardiac transplantation: pathophysiology and management. Curr Opin Nephrol Hyper 1995;4:443-51.

[2] Xue H, Buroski RD, McCarron DA, Bennett WM. Production of contraction in isolated rat aorta by cyclosporin. Transplantation 1987;43(5):715-8.

[3] Yaris E, Tuncer M. Cyclosporin A cremophor-EL augment renal vascular responses to various agonists and nerve stimulation. Arch Int Pharmacol Ther 1995;329(3):405-17.

[4] Golub MS, Lustig S, Berger ME, Lee BND. Altered vascular responses in cyclosporin-treated rats. Transplantation 1989;48(1):1168.

[5] Cartier R, Pearson PJ, Lin PJ, Schaff HV. Time course and extent of recovery of endothelium-dependent contractions and relaxations after direct arterial injury. J Thorac Cardiovasc Surg 1991;102:371-7.

[6] Textor SC, Smith-Powell L, Telles T. Altered pressor responses to $\mathrm{NE}$ and Ang II during cyclosporin A administration to conscious rats. Am J Physiol 1990;258:H854-60.

[7] Auch-Schwelk W, Bossaler C, Gotze S, Thelen J, Fleck E. Endothelial and vascular smooth muscle function after chronic treatment with cyclosporin A. J Cardiovasc Pharmacol 1993;21:435-40.
[8] Roullet JB, Xue H, McCarron DA, Holcomb S, Bennett WM. Vascular mechanisms of cyclosporin-induced hypertension in the rat. $\mathrm{J}$ Clin Invest 1994;93(5):2244-50.

[9] Van den Dopel MA, Van den Meiracker AH, Lameris TW, Boomsma F, Levi M, Weimar W, et al. Cyclosporin A impairs the nocturnal blood pressure fall in renal transplant recipients. Hypertension 1996;28(2):304-7.

[10] Sehested J, Thomas F, Thorn M, Schifter S, Regitz V, Sheikh S, et al. Level and diurnal variations of hormones of interest to the cardiovascular system in patients with heart transplants. Am J Cardiol 1992;69(4):397-402.

[11] Crum R, Fairchild R, Bronsther O, Dominic W, Ward D, Femandez $\mathrm{R}$, et al. Neuroendocrinology of chronic renal failure and renal transplantation. Transplantation 1991;52(5):818-23.

[12] Gerkens JF. Cyclosporin treatment of normal rats produces a rise in blood pressure and decrease renal vascular responses to nerve stimulation, vasoconstrictors and endothelium-dependent dilators. J Pharmacol Exp Ther 1989;250(3):1105-12.

[13] Duruibe VA, Okonmah A, Panton L. Effect of cyclosporin on rat kidney catecholamines. Life Sci 1990;47:255-61.

[14] Reis F, Tavares P, Teixeira F. The distribution of catecholamines between plasma and platelets in cyclosporin A-induced hypertensive rats. Pharmacol Res 2000;41(2):129-35.

[15] Tavares P, Martinez-Salgado C, Eleno N, Teixeira F, Lopez-Novoa JM. Effect of cyclosporin A on rat smooth muscle cell proliferation. J Cardiovasc Pharmacol 1998;31:46-9.

[16] Nashan B, Bleck J, Wonigeit K, Christians U, Sewing KF, Beveridge $\mathrm{T}$, et al. Effect of the application form of cyclosporin on blood levels: comparison of oral solution and capsules. Transplant Proc 1988;20:637-9.

[17] Holt DW, Johnston A, Roberts NB, Tredger JM, Trull AK. Methodological and clinical aspects of cyclosporin monitoring. Report of the association of clinical biochemists task force. Clin Biochem 1994;31:420-46.

[18] Stein CM, He H, Pincus T, Wood AJ. Cyclosporin impairs vasodilatation without increased sympathetic activity in humans. Hypertension 1995;26(4):705-10.

[19] Lamb FS, Webb RC. Cyclosporin augments reactivity of isolated blood vessels. Life Sci 1987;40:2571-8.

[20] Golub MS, Berger ME. Direct augmentation by cyclosporin A of the vascular contractile response to nerve stimulation. Hypertension 1987;9:III96-100.

[21] Johnson RG, Carty SE, Hayflick S, Scarpa A. Mechanism of accumulation of tyramine, metaraminol, and isoproterenol in isolated chromaffin granules and ghosts. Biochem Pharmacol 1982;31:81523.

[22] Pestana M, Vieira-Coelho MA, Pinto-do-Ó PC, Fernandes MH, Soares-da-Silva P. Assessment of renal dopaminergic system activity during cyclosporin $\mathrm{A}$ administration in the rat. $\mathrm{Br} \mathrm{J}$ Pharmacol 1995;115:1349-58.

[23] Zhang W, Victor RG. Calcineurin inhibitors cause renal efferent activation in rats: a novel mechanism of cyclosporin-induced hypertension. Am J Hyper 2000;13(9):999-1004.

[24] Zhang W, Li JL, Hosaka M, Janz R, Shelton JM, Albright GM, et al. Cyclosporin A-induced hypertension involves synapsin in renal sensory nerve endings. Proc Natl Acad Sci USA 2000;97(17):976570. 